\title{
Correction to: Antisocial Personality Disorder and Physical Partner Violence Among Single and Dual Substance-Abusing Couples
}

\author{
Michelle L. Kelley ${ }^{1,2} \cdot$ Abby L. Braitman ${ }^{1}$
}

Published online: 12 May 2018

(C) Springer Science+Business Media, LLC, part of Springer Nature 2018

\section{Correction to: J Fam Viol (2016) 31:423-431}

$$
\text { https://doi.org/10.1007/s10896-016-9802-6 }
$$

The original version of this article unfortunately contained a mistake. There is an error within Table 2. The influence of Female diagnosis of antisocial personality disorder (column 2) on Female perpetration of violence should be listed as $\mathrm{B}=12.41, \mathrm{SE}=5.82$, beta $=0.389, p=.033$. This is a change from a non-significant trend $(p<.10)$ to a significant effect $(p<.05)$. All demonstrated trends $(\mathrm{p}<.10)$ are discussed as clinically meaningful effects, and so the primary conclusions of the paper are unchanged.

The correct table is shown next page.

The online version of the original article can be found at https://doi.org/ 10.1007/s10896-016-9802-6

Michelle L. Kelley

mkelley@odu.edu

1 Department of Psychology, Old Dominion University, Norfolk, VA 23529-0267, USA

2 Virginia Consortium Program in Clinical Psychology, Old Dominion University, Norfolk, VA, USA 
Table 2 Perpetration and victimization by APD diagnosis

\begin{tabular}{|c|c|c|c|c|c|c|}
\hline \multirow[t]{2}{*}{ Outcome } & \multicolumn{3}{|c|}{$\begin{array}{l}\text { Male diagnosis of antisocial } \\
\text { personality disorder }\end{array}$} & \multicolumn{3}{|c|}{$\begin{array}{l}\text { Female diagnosis of antisocial } \\
\text { personality disorder }\end{array}$} \\
\hline & $B(S E)$ & $\beta$ & $p$ & $B(S E)$ & $\beta$ & $p$ \\
\hline \multicolumn{7}{|l|}{ Male perpetration of violence } \\
\hline Antisocial Personality Disorder & $4.88^{*}(1.75)$ & 0.462 & .005 & $-1.08(1.91)$ & -0.909 & .571 \\
\hline Couple Diagnosis & $2.60(1.64)$ & 0.237 & .113 & $-0.31(1.47)$ & -0.028 & .833 \\
\hline APD x Couple & $-5.51 *(2.65)$ & -0.396 & .037 & $2.82(2.83)$ & 0.199 & .320 \\
\hline \multicolumn{7}{|l|}{ Female perpetration of violence } \\
\hline Antisocial Personality Disorder & $11.50 *(5.27)$ & 0.353 & .029 & $12.41(5.82)$ & 0.389 & .033 \\
\hline Couple Diagnosis & $0.52(3.44)$ & 0.015 & .880 & $0.78(4.65)$ & 0.025 & .867 \\
\hline APD x Couple & $-3.64(8.43)$ & -0.085 & .666 & $-10.54(9.43)$ & -0.259 & .263 \\
\hline \multicolumn{7}{|l|}{ Male victim of violence } \\
\hline Antisocial Personality Disorder & $9.56 *(2.80)$ & 0.531 & .001 & $4.11(3.22)$ & 0.208 & .201 \\
\hline Couple Diagnosis & $3.95(2.84)$ & 0.211 & .165 & $0.88(2.46)$ & 0.049 & .720 \\
\hline APD x Couple & $-9.83 *(4.37)$ & -0.414 & .024 & $-3.71(4.88)$ & -0.159 & .447 \\
\hline \multicolumn{7}{|l|}{ Female victim of violence } \\
\hline Antisocial Personality Disorder & $9.94 *(4.87)$ & 0.348 & .041 & $15.44 *(7.11)$ & 0.501 & .030 \\
\hline Couple Diagnosis & $1.24(4.14)$ & 0.042 & .765 & $-0.98(3.56)$ & -0.035 & .783 \\
\hline APD x Couple & $-8.66(7.07)$ & -0.230 & .221 & $-7.89(8.79)$ & -0.217 & .370 \\
\hline
\end{tabular}

Bold text and asterisk after the unstandardized parameter coefficient denote significance at the $p<.05$ level. Note that $S E$ refers to empirical bootstrapped standard errors 